\title{
New insights into the heat of adsorption of water, acetonitrile, and $n$-hexane in porous carbon with oxygen functional groups
}

\author{
Chiharu Urita ${ }^{1}$, Koki Urita $^{1 *}$, Takuya Araki ${ }^{1}$, Keiji Horio $^{2}$, Masayuki Yoshida ${ }^{2}$, Isamu Moriguchi ${ }^{1}$ \\ ${ }^{a}$ Graduate School of Engineering, Nagasaki University, 1-14 Bunkyo-machi, Nagasaki 852-8521, \\ Japan \\ ${ }^{\mathrm{b}}$ MicrotracBEL Corp., 8-2-52 Nanko-Higashi, Suminoe-ku, Osaka 559-0031, Japan
}

\author{
*Corresponding author. \\ E-mail address: urita@nagasaki-u.ac.jp (K. Urita)
}

Tel \& fax: +81-95-819-2668

\begin{abstract}
Isosteric heat of adsorption is exquisitely sensitive to structural changes in carbon surfaces based on the energetic behavior of the interactions between adsorbates and carbon materials. We discuss the relationships between porous structures, oxygen functional groups, and heat of adsorption based on the behavior of the heat of adsorption of polar and non-polar fluids on porous carbon materials with oxygen functional groups. The porosity and functional groups of porous carbon materials were estimated from $\mathrm{N}_{2}$ adsorption isotherms at $77 \mathrm{~K}$ and temperature-programmed desorption. High-resolution adsorption isotherms of water, acetonitrile (polar fluid), and $n$-hexane (non-polar fluid) were measured on porous carbon materials with different pore size distributions and amounts of oxygen functional groups at various temperatures. The heats of adsorption were determined by applying the Clausius-Clapeyron equation to the adsorption isotherms. The heat of adsorption curves directly reflect the effects of interactions of fluid-oxygen functional groups, fluidbasal planes of pore walls, and fluid-fluid interfaces. In particular, the heat of adsorption curve of water is very sensitive to surface oxygen functional groups. This finding indicates the possibility of estimating the relative amounts of oxygen functional groups on porous carbon materials based on the amounts of water adsorbed at specific relative pressures.
\end{abstract}

Keywords: Isosteric heat of adsorption, Temperature-programmed desorption, Porous carbon, Water adsorption, Acetonitrile adsorption, Hexane adsorption

Abbreviations: PSD, pore size distribution; HR-TEM, high-resolution transmission electron microscopy; GCMC, grand canonical Monte Carlo; NLDFT, non-local density functional theory; SSA, 
specific surface area; TPD, temperature-programmed desorption

\section{Introduction}

Porous carbon materials have received significant attention as adsorbents [1-3], catalyst-related materials [4-6], and electrode materials [7-10] based on their high surface area, large pore volume, and ease of synthesis. Carbon materials are typically subjected to thermal treatment to add surface functionality (e.g., heteroatom incorporated carbon materials [11-14] and porous carbon materials with high specific surface area [15-18]). Thermal and chemical treatments can easily deform the surface morphologies of carbon materials. Because material performance is significantly affected by the surface structures on which guest molecules or ions interact, information regarding surface structure and porosity is crucial for designing ideal porous carbon materials.

The gas adsorption technique is one useful method for understanding the surface states of carbon materials. Isosteric heat of adsorption is calculated by applying the Clausius-Clapeyron equation [1921] to adsorption isotherms measured at two or more different temperatures. The obtained heat of adsorption curve (heat curve) versus the amount of adsorbed gas accurately represents the interactions between adsorbates and solid surfaces [22, 23]. Fan et al. reported that a sharp specific change called a heat spike can be observed in the heat curve of $\mathrm{N}_{2}$ adsorption on a homogenous carbon surface, such as graphite [24]. When adsorbed molecules close to the surface interact with neighboring molecules on the first layer of a carbon material, all adsorbed molecules are reconstructed and a heat spike appears as a result of the phase change associated with drastic heat release [23]. Heat of adsorption has been studied extensively for nonporous carbon materials with relatively flat surfaces and it has been determined that heat of adsorption represents various interactions, such as fluid-fluid, fluidfunctional group, and fluid-basal plane interactions. In the case of porous carbon materials, the contribution of pores to heat of adsorption should be also considered. However, the complexity of this issue has hindered the understanding of the heat of adsorption behavior of porous carbon materials with oxygen functional groups and few studies have linked this behavior to porous carbon structures.

In this paper, we discuss the influence of pore size distributions (PSDs) and the amount of surface functional groups on the isosteric heat of adsorption of polar and non-polar adsorbents. We tested three types of porous carbon materials. Two of the tested carbon materials have similar PSDs, but different amounts of oxygen functional groups. The third material has a different PSD compared to the other two materials. A measurement system with extremely low air leakage was used to derive accurate adsorption isotherms. Adsorption isotherms captured at a low relative pressure enabled us to determine the PSDs for an average pore size of $\sim 0.35 \mathrm{~nm}$ and the heat of adsorption based on an initial 
adsorption state.

\section{Experimental section}

The porous carbon samples used in this study were activated using an alkali metal salt solution (MC Evolve Technologies Co.). Here, we denote the samples as $\mathrm{M} X(X=1 \ldots 3)$. M1 and M2 were produced through the $\mathrm{KOH}$ activation of phenolic resin and petroleum coke, respectively. M3 was produced through a similar $\mathrm{KOH}$ activation treatment of petroleum coke [25]. Sample M3 had been stored in an ambient atmosphere for 25 years, meaning its surface should have been fully oxidized. The carbon framework pores were observed directly using a high-resolution scanning transmission electron microscope (ARM-200CF, JEOL Ltd) in transmission mode at an acceleration voltage of 120 $\mathrm{kV}$. The sample porosities were determined from $\mathrm{N}_{2}$ adsorption isotherms at $77 \mathrm{~K}$ (BELSORP-max, MicrotracBEL Corp.). The $\mathrm{N}_{2}$ adsorption isotherms were carefully measured at a relative pressure $\left(p / p_{0}\right)$ on the order of $10^{-8}$, corresponding to a pore size of $\sim 0.35 \mathrm{~nm}$, following pretreatment under vacuum at $423 \mathrm{~K}$ for $5 \mathrm{~h}$. We identified pretreatment conditions that were suitable for pore structure analysis based on the $\mathrm{N}_{2}$ adsorption isotherms of the activated carbon materials. The amounts of oxygen functional groups were estimated using temperature-programmed desorption (TPD; BELCATII, MicrotracBEL Corp.) combined with mass spectrometry (BELMass, MicrotracBEL Corp.). Approximately $500 \mathrm{mg}$ of porous carbon was heated from room temperature to $1273 \mathrm{~K}$ at a heating rate of $10 \mathrm{~K} / \mathrm{min}$ under a He flow rate of $30 \mathrm{ml} / \mathrm{min}$. The isosteric heats of adsorption $\left(Q_{s t}\right)$ of water, acetonitrile, and $n$-hexane were calculated by applying the Clausius-Clapeyron equation to the adsorption isotherms of each vapor measured at 293, 298, and $303 \mathrm{~K}$ (BELSORP-maxII, MicrotracBEL Corp.). The Snyder polarity indexes of water, acetonitrile, and $n$-hexane are 9, 6.2, and 0 , respectively [26]. The carbon samples were pretreated under vacuum at $423 \mathrm{~K}$ for $18 \mathrm{~h}$. We chose a longer pretreatment time for this step because there was a possibility of water molecules entering deeper, smaller pores. The adsorbate liquids were degassed through freeze-pump-thaw cycles in advance.

\section{Results and Discussion}

The pore shapes of the carbon samples are clearly visible in the high-resolution transmission electron microscopy (HR-TEM) images in Fig. 1. The carbon samples have similar shapes of carbon frameworks with porous structures in which cage-shaped pores are interconnected. Detailed porosities were estimated from the $\mathrm{N}_{2}$ adsorption isotherms at $77 \mathrm{~K}$ (Fig. 2a). A single logarithmic plot of the $\mathrm{N}_{2}$ adsorption isotherms presented in Fig. $2 \mathrm{~b}$ focuses on the behavior of $\mathrm{N}_{2}$ adsorption into the micropores. 
One can see that sample M1 has a larger number of micropores compared to the other samples. Fig. 2c presents the PSDs calculated by applying the grand canonical Monte Carlo (GCMC) method [27] with a standard slit pore model. The GCMC PSDs were obtained using BELMaster ${ }^{\mathrm{TM}}$ software (MicrotracBEL Corp.). We also performed this calculation using the non-local density functional theory (NLDFT) method with a heterogeneous carbon surface model [28, 29] (supplemental Fig. S1) by using a demo version of SAIEUS software (Micromeritics Instrument Corp., www.nldft.com/download/). The PSDs obtained by both methods indicate that sample M1 is more microporous than samples M2 and M3, and that samples M2 and M3 have similar PSDs. Sample M1 is a microporous carbon material with a pore size smaller than $2 \mathrm{~nm}$, where the micropore volume accounts for more than $90 \%$ of total pore volume. Samples M2 and M3 have similar PSDs, where the dominant pore size ranges from 1 to $3 \mathrm{~nm}$ and $\sim 60 \%$ of the pore volume consists of micropores. Table 1 lists the specific surface areas (SSAs) and total pore volumes obtained by the GCMC method. The total pore volumes were calculated directly from the adsorption isotherms. The total pore volumes correspond reasonably well with the results of the GCMC calculations. Additionally, each porous carbon material has a relatively large SSA and pore volume.
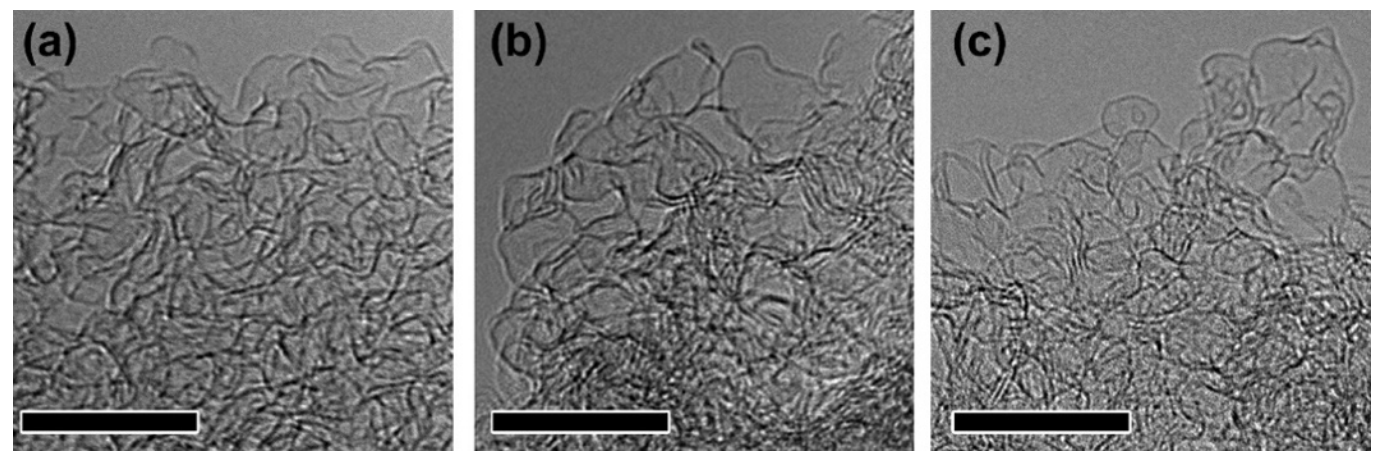

Fig. 1 HR-TEM images of samples (a) M1, (b) M2, and (c) M3. Scale bar: $10 \mathrm{~nm}$. 
(a)

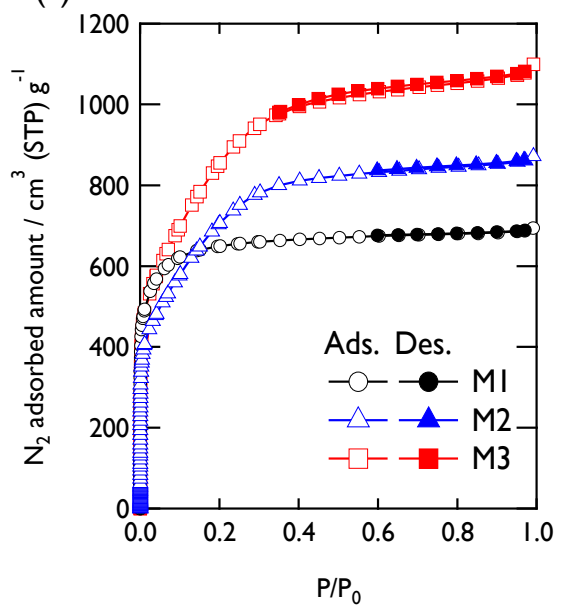

(b)

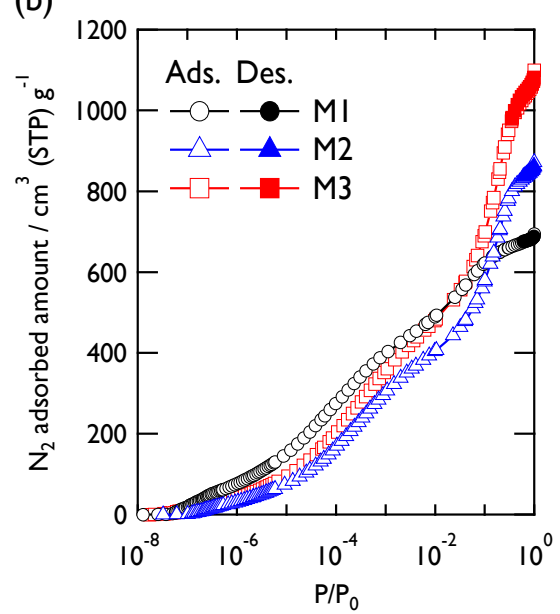

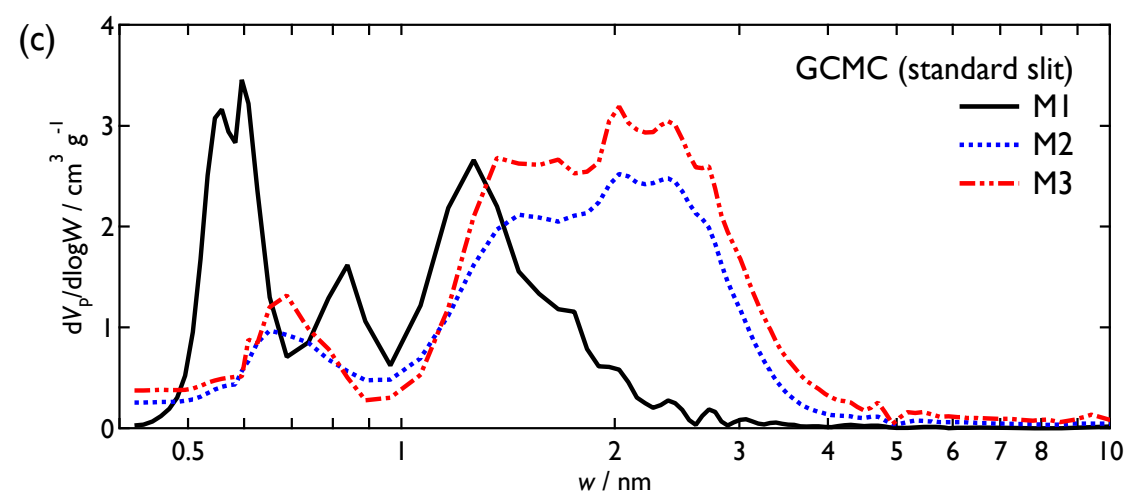

Fig. 2 (a) $\mathrm{N}_{2}$ adsorption isotherms for samples M1 (black circle), M2 (blue triangle), and M3 (red square) at 77 K. (b) Single logarithmic plot of (a). Open and closed markers are adsorption and desorption branches, respectively. (c) Pore size distribution of samples M1 (black solid line), M2 (blue dotted line), and M3 (red dashed line), calculated using the GCMC method.

Table 1 Porosity of porous carbon samples determined by the GCMC method* and adsorbed volume at $p / p_{0}=0.99^{* *}$.

\begin{tabular}{cccc}
\hline & $\begin{array}{c}\text { SSA* } \\
\left(\mathrm{m}^{2} / \mathrm{g}\right)\end{array}$ & $\begin{array}{c}\text { Total pore volume* } \\
\left(\mathrm{cm}^{3} / \mathrm{g}\right)\end{array}$ & $\begin{array}{c}\text { Total pore volume**} \\
\left(\mathrm{cm}^{3} / \mathrm{g}\right)\end{array}$ \\
\hline M1 & 1623 & 1.02 & 1.07 \\
M2 & 1321 & 1.22 & 1.35 \\
M3 & 1611 & 1.55 & 1.70 \\
\hline
\end{tabular}

TPD curves quantitatively represent the gas evolution derived from oxygen functional groups (Fig. 3). $\mathrm{H}_{2} \mathrm{O}$ evolution is attributed to the dehydration of either neighboring carboxylic groups with the 
formation of a carboxyl anhydride group or to that of ortho-dihydroquinone-type phenolic hydroxyl groups [30,31]. Evolved CO stems from acid anhydride, ether, hydroxyl, quinone, and carbonyl, and the $\mathrm{CO}_{2}$ stems from acid anhydride, carboxyl, and lactone [32, 33]. The TPD curves were deconvoluted based on the specific desorption temperature of each oxygen group. The evolution amounts of $\mathrm{CO}$ and $\mathrm{CO}_{2}$ calculated from the integration of the deconvoluted curves are presented in Fig. 3. Specific values are summarized in the supplemental information (Table S1). The amount of $\mathrm{CO}$ is much greater than that of $\mathrm{CO}_{2}$. This is because the oxygen functional groups (anhydride, ether, hydroxyl, quinone, and carbonyl) related to $\mathrm{CO}$ evolution are present in relatively large amounts on the porous carbon materials (Fig. 4). In particular, acid anhydride should be a dominant functional group because the $\mathrm{CO}$ and $\mathrm{CO}_{2}$ evolutions for all samples are largely derived from acid anhydride and lactone, respectively. The amounts of oxygen functional groups were the same for samples M1 and M2. Sample M3 has the largest total amount of surface oxygen groups with a value approximately three times larger than those of the other samples. Therefore, sample M3 is an oxygen-functionalgroup-rich porous carbon material. As shown in Fig. 1, samples M2 and M3 have similar PSDs, which are different from the PSD of M1. The porous carbon samples were classified based on their PSDs and amounts of oxygen functional groups. Samples M1 and M2 have similar amounts of oxygen functional groups, but different PSDs, whereas samples M2 and M3 have similar PSDs, but different amounts of oxygen functional groups is.
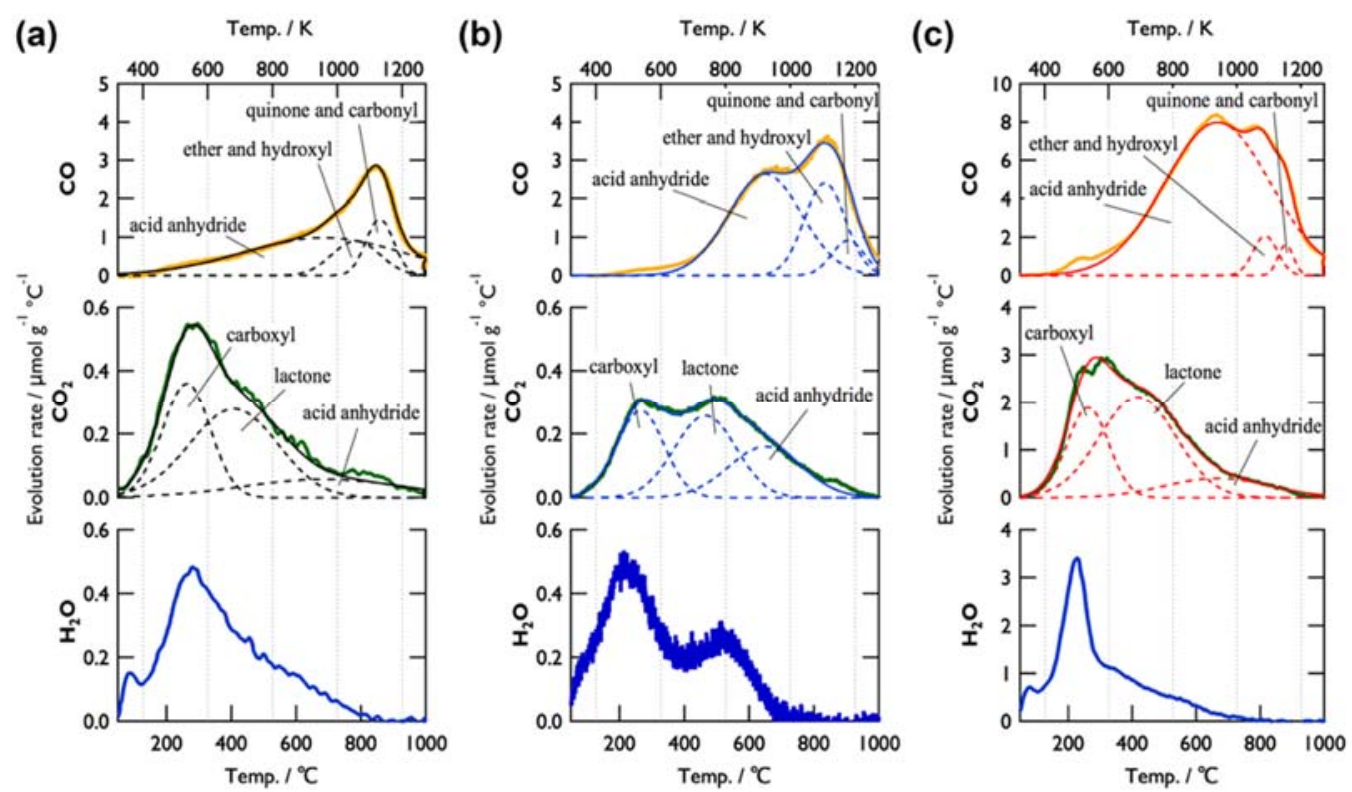

Fig. 3 TPD curves of samples (a) M1, (b) M2, and (c) M3 corresponding to $\mathrm{CO}$ (top), $\mathrm{CO}_{2}$ (middle), and $\mathrm{H}_{2} \mathrm{O}$ (bottom) desorption. Broken lines represent the deconvoluted curves of contributing surface oxygen functional groups. 
(a)

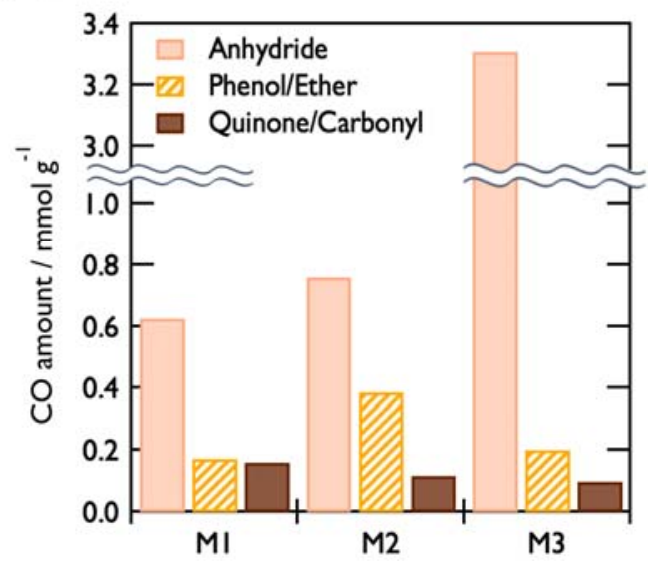

(b)

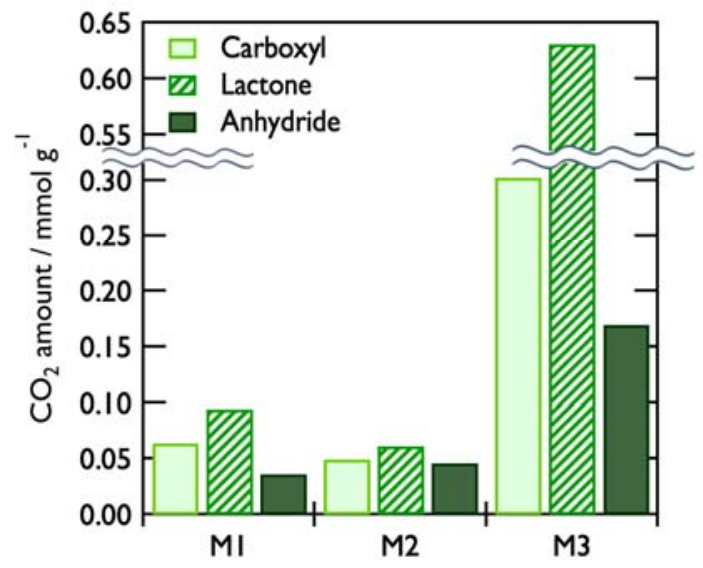

Fig. 4 Amounts of (a) $\mathrm{CO}$ and (b) $\mathrm{CO}_{2}$ derived from the oxygen functional groups on each porous carbon material.

Fig. 5 presents the vapor adsorption isotherms at $298 \mathrm{~K}$ and $Q_{\text {st }}$ for water, acetonitrile, and $n$-hexane for samples M1, M2, and M3. All of the adsorption isotherms at 293, 298, and $303 \mathrm{~K}$ are presented in the supplemental information (Figs. S2-S4). The horizontal axes in Fig. 5 are shown at a logarithmic scale to represent the initial adsorption states. Vapor adsorption isotherms and heat curves shown at a linear scale are presented in the supplemental information (Fig. S5). $Q_{\text {st }}$ at each relative pressure was determined from the slope of the following equation:

$=-\frac{Q_{s t}}{R T}$

$+C$

where $P$ is the respective absolute pressure, $R$ is the gas constant, $T$ is the adsorption temperature (293, 298 , and $303 \mathrm{~K})$ and $C$ is the integral constant. The coefficient of determination $\left(R^{2}\right)$ for $Q_{\mathrm{st}}$ calculation is presented in Fig. 5 (top row) and represents the accuracy of the value. We considered $Q_{\text {st }}$ changes versus the relative pressure with an $R^{2}$ value over 0.9 . The relative pressure range of $10^{-3}-0.4$ on the water adsorption isotherm corresponds to the initial adsorption state up to the adsorption into micropores, whose adsorption isotherm (see the supplemental information, Fig. S6) is presented as a type-V isotherm using the International Union of Pure and Applied Chemistry (IUPAC) classification [34]. The amount of water adsorbed increases dramatically at $p / p_{0}=0.5$ in Fig. S6. The increasing trend at and above this relative pressure corresponds micropore filling [35], meaning the water 
adsorption at the relative pressure range presented in Fig. 5 should reflect the adsorption to a specific site or ultramicropore. The largest water adsorption occurred for sample M3, which has a relatively large amount of oxygen functional groups. Although the PSD of sample M2 is similar to that of sample M3, the amount of water adsorbed by sample M2 is the same as that adsorbed by sample M1. $Q_{\text {st }}$ changes clearly represent the adsorption behaviors of porous carbon materials with oxygen functional groups. Water adsorption occurs according to the following steps: (1) direct interaction of water molecules with functional groups, (2) growth of water clusters on functional groups, and (3) adsorption of water on either the boundaries of clusters or between neighboring clusters [23]. The large $Q_{\text {st }}$ value of sample M3 reflects strong fluid-functional group interactions .

The adsorption isotherms of acetonitrile and $n$-hexane are type-I isotherms according to the IUPAC classification. Their adsorption amounts roughly correspond to their PSDs, except for the range of relative pressures below $\sim 10^{-4}$. The adsorption amounts for samples M2 and M3, which have relatively large pores, increase at high relative pressures compared to sample M1. However, the acetonitrile adsorption of M3 does not reflect its PSD at low relative pressures, where the acetonitrile adsorption isotherm differs significantly from the $n$-hexane adsorption isotherm. Based on their Snyder polarity indexes, acetonitrile and $n$-hexane behave as polar and non-polar molecules, respectively. Because the non-polar $n$-hexane has little interaction with oxygen functional groups, PSD is considered to have a significant influence on the adsorption isotherm. At low pressures, the amount of adsorbed $n$-hexane for sample M1 is higher than that for samples M2 and M3. The $Q_{\text {st }}$ value of $n$-hexane for sample M1 is also higher than that for samples M2 and M3. This reflects the higher adsorption potential of the microporous sample M1 compared to the lower adsorption potentials of the mesoporous samples M2 and M3. The $Q_{\text {st }}$ value of $n$-hexane adsorption for sample M1 shows an upward deviation after $p / p_{0}=$ 0.05 and does not appear to reflect the real value based on the difficulty of calculation using the C-C equation in the range where the amount of $n$-hexane adsorbed for sample M1 does not increase. In contrast, the $Q_{\text {st }}$ values of $n$-hexane adsorption for samples M2 and M3 decrease to the heat of condensation. This indicates that the adsorbed state of $n$-hexane on mesopores approaches the liquid state. The high $Q_{\text {st }}$ value of initial adsorption for $n$-hexane without adsorption onto oxygen functional groups is attributed to the interaction of the fluid with basal planes, which are pore surfaces. The $Q_{\text {st }}$ value eventually reaches the heat of condensation released by fluid-fluid interactions because further increases in relative pressure lead to multi-layer adsorption.

Although both acetonitrile and water are polar molecules, the features of adsorption of acetonitrile are distinct from those of water. The interaction between water and basal planes is negligibly small compared to the fluid-functional group interactions [36]. Acetonitrile with a methyl group should 
strongly interact with basal planes. The $Q_{\text {st }}$ values of samples M1 and M2, which have relatively low number of functional groups, represent the effects of fluid-basal plane interactions at low relative pressures. In contrast, the $Q_{\text {st }}$ value of sample M3 at a low relative pressure is lower than the heat of condensation because fluid-functional group interactions are dominant. Therefore, acetonitrile adsorption proceeds with simultaneous adsorption on functional groups and the basal planes of pores.

Fig. 6 presents the amounts of $\mathrm{CO}_{2}$ evolved from carboxyl, lactone, and acid anhydride on the samples, as well as the amount of water adsorbed at the minimum $Q_{\text {st. }}$ The $Q_{\text {st }}$ values of all samples reach minimums at a relative pressure of approximately $10^{-2}$. Because the amount of water adsorbed is assumed to correspond to the amount of surface oxygen functional groups, the relative amount of oxygen functional groups should be in the order of sample M2 < sample M1 < sample M3. This tendency matches the changes in the amounts of carboxyl and lactone on the samples. This result suggests that the relative amount of adsorbed water at a relative pressure of $10^{-2}$, where $Q_{\mathrm{st}}$ is minimized, is considered to be an index for the amount of hydrophilic carboxyl and lactone in porous carbon materials. These results correspond to the studies by Liu et al. [37], Puri et al. [38, 39], Vartapetyan and Voloshchuk [40], Fletcher et al. [41], and Xiao et al. [42].

(a) Water

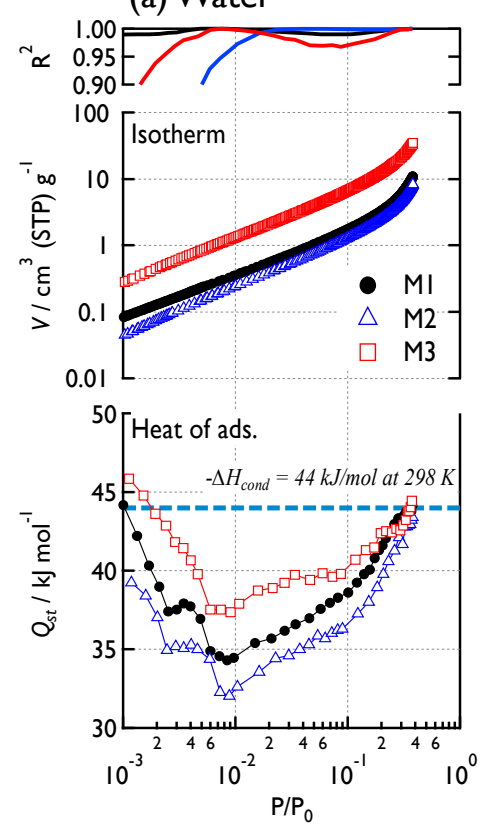

(b) Acetonitrile
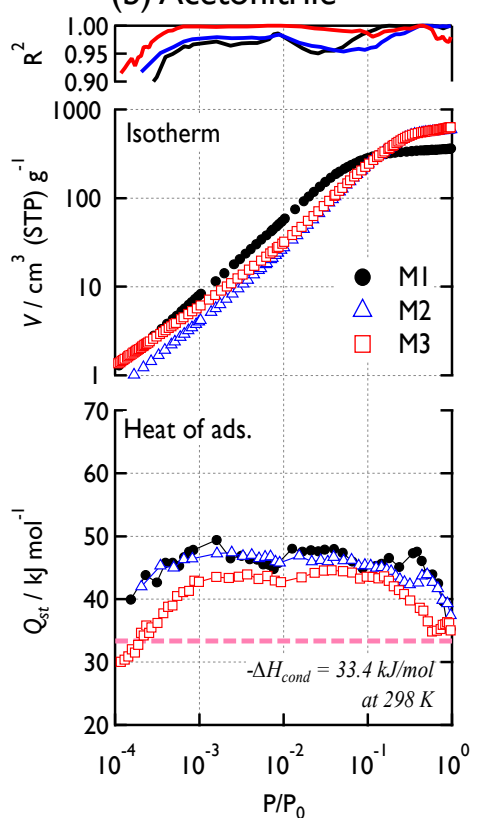

(c) Hexane

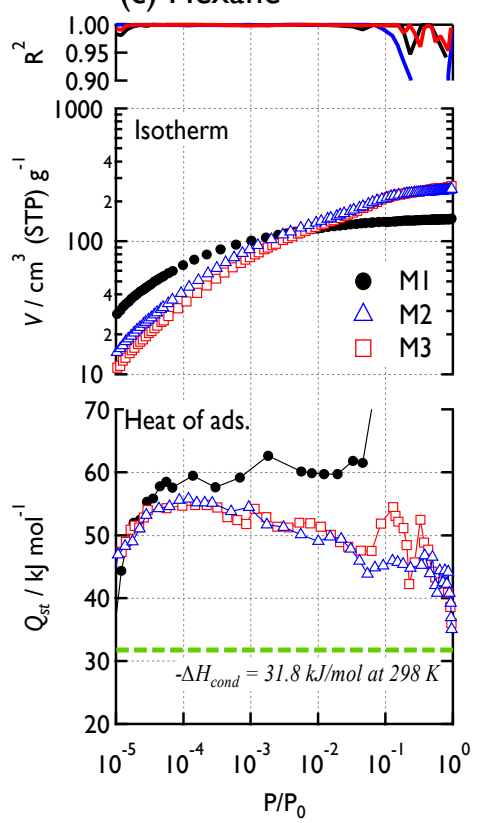

Fig. 5 Vapor adsorption isotherms at $298 \mathrm{~K}$ (middle), isosteric heat of adsorption (bottom), and coefficient of determination $\left(R^{2}\right)$ for applying the Clausius-Clapeyron equation (top) to samples M1 (black closed circle), M2 (blue open triangle), and M3 (red open square) for different adsorbents: (a) water, (b) acetonitrile, and (c) $n$-hexane. Broken lines in the bottom row represent the heat of 
condensation of each adsorbent.

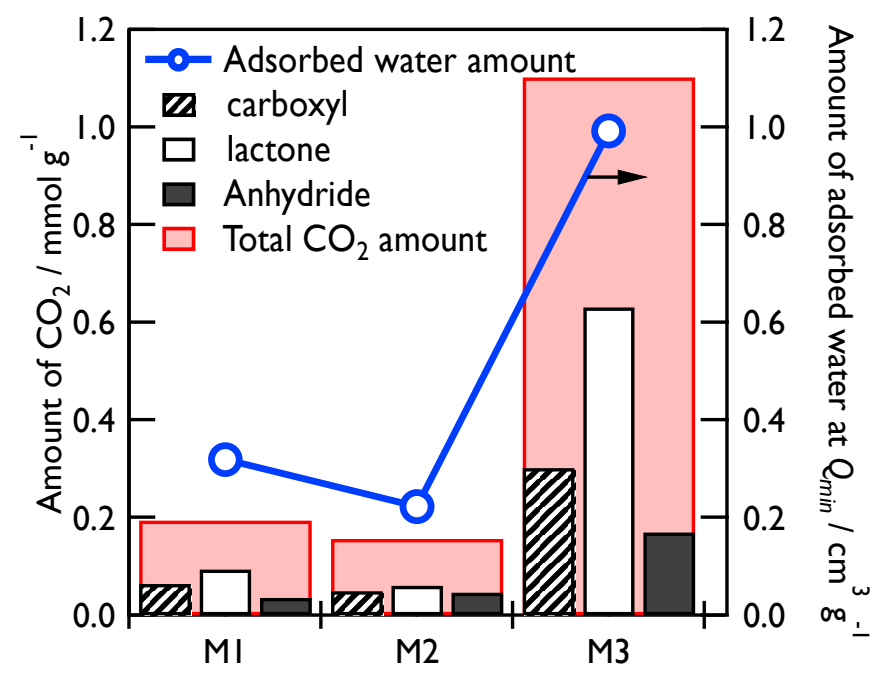

Fig. 6 Comparison of amounts of $\mathrm{CO}_{2}$ evolved from carboxyl and lactone with the amount of adsorbed water at the minimum value of $Q_{\mathrm{st}}$ for samples M1, M2, and M3.

\section{Conclusion}

The isosteric heat of adsorption of polar (water and acetonitrile) and non-polar ( $n$-hexane) fluids for porous carbon materials with oxygen functional groups was carefully examined at low relative pressures. The heat curves of $n$-hexane for porous carbon materials directly reflected the porous structures because non-polar fluids are insensitive to the oxygen functional groups on porous carbon materials. Therefore, porous carbon materials with accessible pores exhibit a decrease in heat of adsorption toward the heat of condensation based on the effects of fluid-fluid interactions. In contrast, the heat curve behavior of polar fluids differs based on their affinity with the basal planes of carbon surfaces. The heat curves of polar fluids with relatively high affinities, such as acetonitrile, complicate the understanding of the interactions of fluids with porous carbon materials because adsorption processes on oxygen functional groups and pores proceed simultaneously at measurable relative pressures. However, the heat curve of water, which is a polar fluid with low affinity with basal planes, strongly reflects the amount of hydrophilic functional groups, even for adsorption on porous carbon materials. Furthermore, the amount of adsorbed water at a relative pressure of $10^{-2}$ corresponds to the minimum $Q_{\text {st }}$ value, meaning the amount adsorbed correlates strongly with the amount of hydrophilic functional groups on porous carbon materials. These results clearly indicate that non-polar molecules can serve as helpful probes for understanding carbon porosity. Additionally, water is a preferable 
probe molecule for estimating the relative amount of surface oxygen on both non-porous and porous carbon materials.

\section{Acknowledgement}

This work was partially supported by JSPS KAKENHI Grant No. 16H05967.

\section{References}

[1] M. Olivares-Marín, M.M. Maroto-Valer, Greenh. Gases 2 (2012) 20.

[2] S. Porada, R. Zhao, A. van der Wal, V. Presser, P.M. Biesheuvel, Prog. Mater. Sci. 58 (2013) 1388.

[3] J. Wang, I. Senkovska, S. Kaskel, Q. Liu, Carbon 75 (2014) 372.

[4] F. Rodriguez-Reinoso, J. de Dios López-González, C. Moreno-Castilla, A. Guerrero-Ruiz, I. RodriguezRamos, Fuel 63 (1984) 1089.

[5] S.H. Joo, S.J. Choi, I. Oh, J. Kwak, Z. Liu, O. Terasaki, R. Ryoo, Nature 412 (2001) 169.

[6] I. Matos, M. Bernardo, I. Fonseca, Catal. Today 285 (2017) 194.

[7] E. Frackowiak, F. Béguin, Carbon 39 (2001) 937.

[8] J. Chmiola, G. Yushin, Y. Gogotsi, C. Portet, P. Simon, P.L. Taberna, Science 313 (2006) 1760.

[9] S. Oro, K. Urita, I. Moriguchi, Chem. Commun. 50 (2014) 7143.

[10] K. Urita, C. Urita, K. Fujita, K. Horio, M. Yoshida, I. Moriguchi, Nanoscale 9 (2017) 15643.

[11] S.-B. Choi, B.-K. Kim, P. Boudjouk, D.G. Grier, J. Am. Chem. Soc. 123 (2001) 8117.

[12] M. Wu, Q. Zha, J. Qiu, Y. Guo, H. Shang, A. Yuan, Carbon 42 (2004) 205.

[13] J.P. Paraknowitsch, A. Thomas, Energy Environ. Sci. 6 (2013) 2839.

[14] H.-W. Liang, X. Zhuang, S. Brüller, X. Feng, K. Müllen, Nat. Commun. 5 (2014) 4973.

[15] J. Miyamoto, H. Kanoh, K. Kaneko, Carbon 43 (2005) 855.

[16] Y. Gogotsi, A. Nikitin, H. Ye, W. Zhou, J.E. Fischer, B. Yi, H.C. Foley, M.W. Barsoum, Nat Mater 2 (2003) 591.

[17] Y. Xia, Z. Yang, R. Mokaya, Nanoscale 2 (2010) 639.

[18] J. Wang, S. Kaskel, J. Mater. Chem. 22 (2012) 23710.

[19] L.G. Joyner, P.H. Emmett, J. Am. Chem. Soc. 70 (1948) 2353.

[20] C. Pierce, R.N. Smith, J. Phys. Colloid Chem. 54 (1950) 354.

[21] H. Pan, J.A. Ritter, P.B. Balbuena, Langmuir 14 (1998) 6323.

[22] Y. Grillet, F. Rouquerol, K. Rouquerol, J. Colloid Interface Sci. 70 (1979) 239.

[23] T. Horikawa, Y. Zeng, D.D. Do, K.-I. Sotowa, J.R. Alcántara Avila, J. Colloid Interface Sci. 439 (2015) 1. 
[24] C. Fan, G. Birkett, D.D. Do, J. Colloid Interface Sci. 342 (2010) 485.

[25] A.N. Wennerberg, T.M. O'Grady, Active carbon process and composition, Standard Oil Company, US, 1978.

[26] L.R. Snyder, J. Chromatogr. A 92 (1974) 223.

[27] M.T. Miyahara, R. Numaguchi, T. Hiratsuka, K. Nakai, H. Tanaka, Adsorption 20 (2013) 213.

[28] J. Jagiello, J.P. Olivier, Carbon 55 (2013) 70.

[29] J. Jagiello, J.P. Olivier, Adsorption 19 (2013) 777.

[30] J. Surygała, R. Wandas, E. Śliwka, Fuel 72 (1993) 409.

[31] S. Haydar, C. Moreno-Castilla, M.A. Ferro-García, F. Carrasco-Marín, J. Rivera-Utrilla, A. Perrard, J.P. Joly, Carbon 38 (2000) 1297.

[32] F. Rodríguez-Reinoso, M. Molina-Sabio, Adv. Colloid Interface Sci. 76-77 (1998) 271.

[33] J.L. Figueiredo, M.F.R. Pereira, M.M.A. Freitas, J.J.M. Órfão, Carbon 37 (1999) 1379.

[34] M. Thommes, K. Kaneko, A.V. Neimark, J.P. Olivier, F. Rodriguez-Reinoso, J. Rouquerol, K.S.W. Sing, Pure Appl. Chem. 87 (2015).

[35] T. Ohba, K. Kaneko, J. Phys. Chem. C 111 (2007) 6207.

[36] D.D. Do, S.L. Johnathan Tan, Y. Zeng, C. Fan, V.T. Nguyen, T. Horikawa, D. Nicholson, J. Colloid Interface Sci. 446 (2015) 98.

[37] L. Liu, S. Tan, T. Horikawa, D.D. Do, D. Nicholson, J. Liu, Adv. Colloid Interface Sci. 250 (2017) 64.

[38] B.R. Puri, K. Murari, D.D. Singh, J. Phys. Chem. 65 (1961) 37.

[39] B.R. Puri, Carbon 4 (1966) 391.

[40] R.S. Vartapetyan, A.M. Voloshchuk, Russ. Chem. Rev. 64 (1995) 985.

[41] A.J. Fletcher, Y. Uygur, K.M. Thomas, J. Phys. Chem. C 111 (2007) 8349.

[42] J. Xiao, Z. Liu, K. Kim, Y. Chen, J. Yan, Z. Li, W. Wang, J. Phys. Chem. C 117 (2013) 23057. 
Supplemental information for

New insights into the heat of adsorption of water, acetonitrile and $\boldsymbol{n}$-hexane in porous carbon with oxygen functional groups

Chiharu Urita $^{1}$, Koki Urita ${ }^{1 *}$, Takuya Araki ${ }^{1}$, Keiji Horio $^{2}$, Masayuki Yoshida ${ }^{2}$, Isamu Moriguchi ${ }^{1}$

${ }^{a}$ Graduate School of Engineering, Nagasaki University, 1-14 Bunkyo-machi, Nagasaki 852-8521, Japan

${ }^{\mathrm{b}}$ MicrotracBEL Corp., 8-2-52 Nanko-Higashi, Suminoe-ku, Osaka 559-0031, Japan

*Corresponding author: K. Urita (urita@nagasaki-u.ac.jp) 

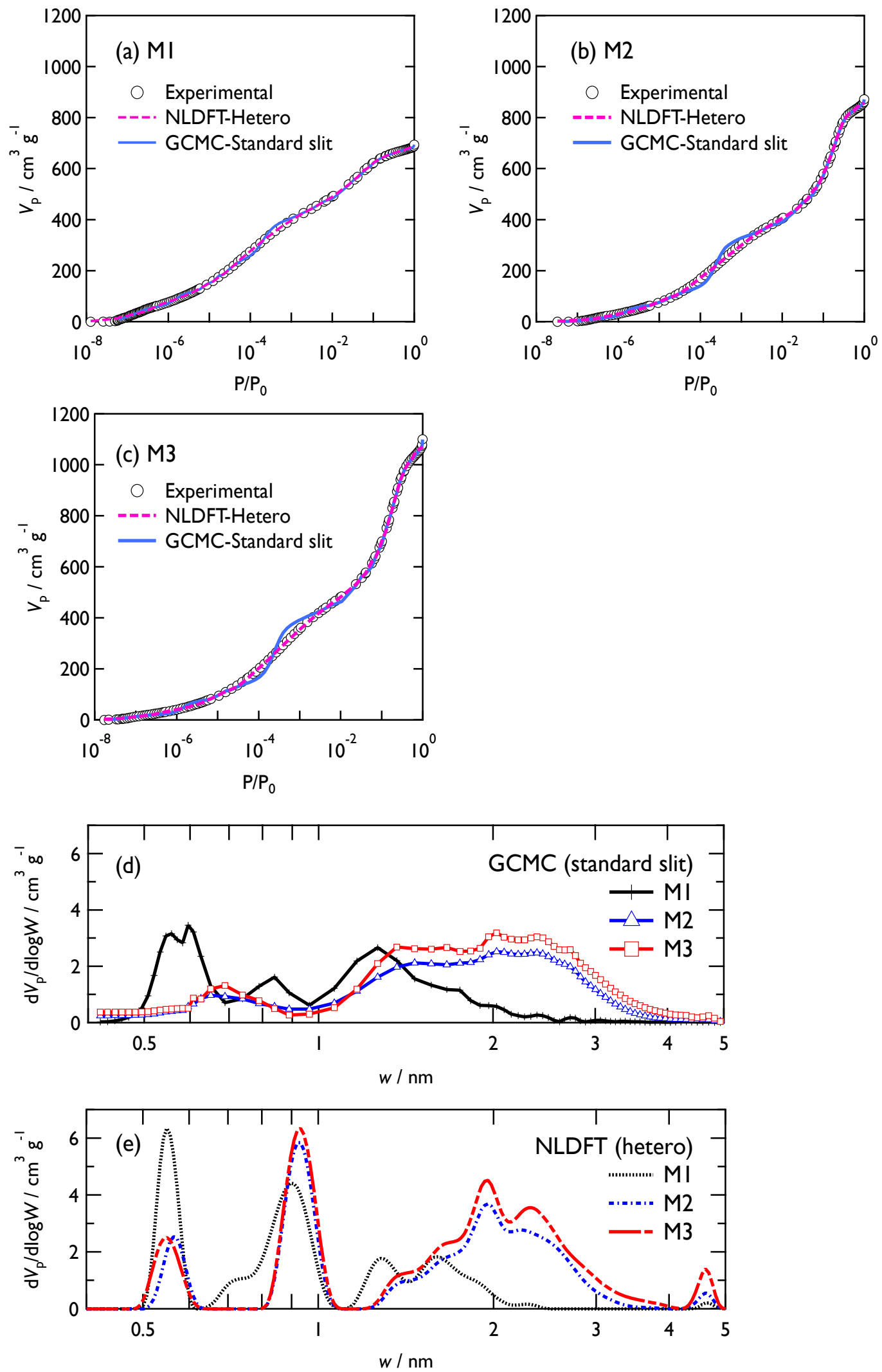

Fig. S1 $\mathrm{N}_{2}$ adsorption isotherms at $77 \mathrm{~K}$ obtained by the NLDFT method (heterogeneous carbon surface) and the GCMC method (standard slit) of samples (a) M1, (b) M2, and (c) M3. Experimental (circle), NLDFT-heterogeneous surface (dashed line) and GCMC-standard slit (solid line). Pore size distributions calculated using the GCMC method (d) and the NLDFT method (e). For the NLDFT calculation, we used the regularization parameter $\lambda$ giving the maximum curvature of the L-curve. 
Table S1 Amounts of evolved gases and the related functional groups on each porous carbon material.

\begin{tabular}{llccc}
\hline Gas & $\left(\mathbf{m m o l ~}^{-1}\right)$ & M1 & M2 & M3 \\
\hline $\mathbf{C O}$ & Total amount of CO & 0.957 & 1.265 & 3.606 \\
& Acid anhydride & 0.625 & 0.760 & 3.309 \\
& Ether and hydroxyl & 0.171 & 0.387 & 0.200 \\
& Quinone and carbonyl & 0.161 & 0.118 & 0.097 \\
\hline $\mathbf{C O}_{2}$ & Total amount of $\mathrm{CO}_{2}$ & 0.194 & 0.156 & 1.103 \\
& Carboxyl & 0.064 & 0.049 & 0.302 \\
& Lactone & 0.094 & 0.061 & 0.631 \\
& Acid anhydride & 0.036 & 0.046 & 0.170 \\
\hline $\mathbf{H}_{\mathbf{2}} \mathbf{O}$ & Total amount & 0.198 & 0.202 & 0.560 \\
\hline
\end{tabular}



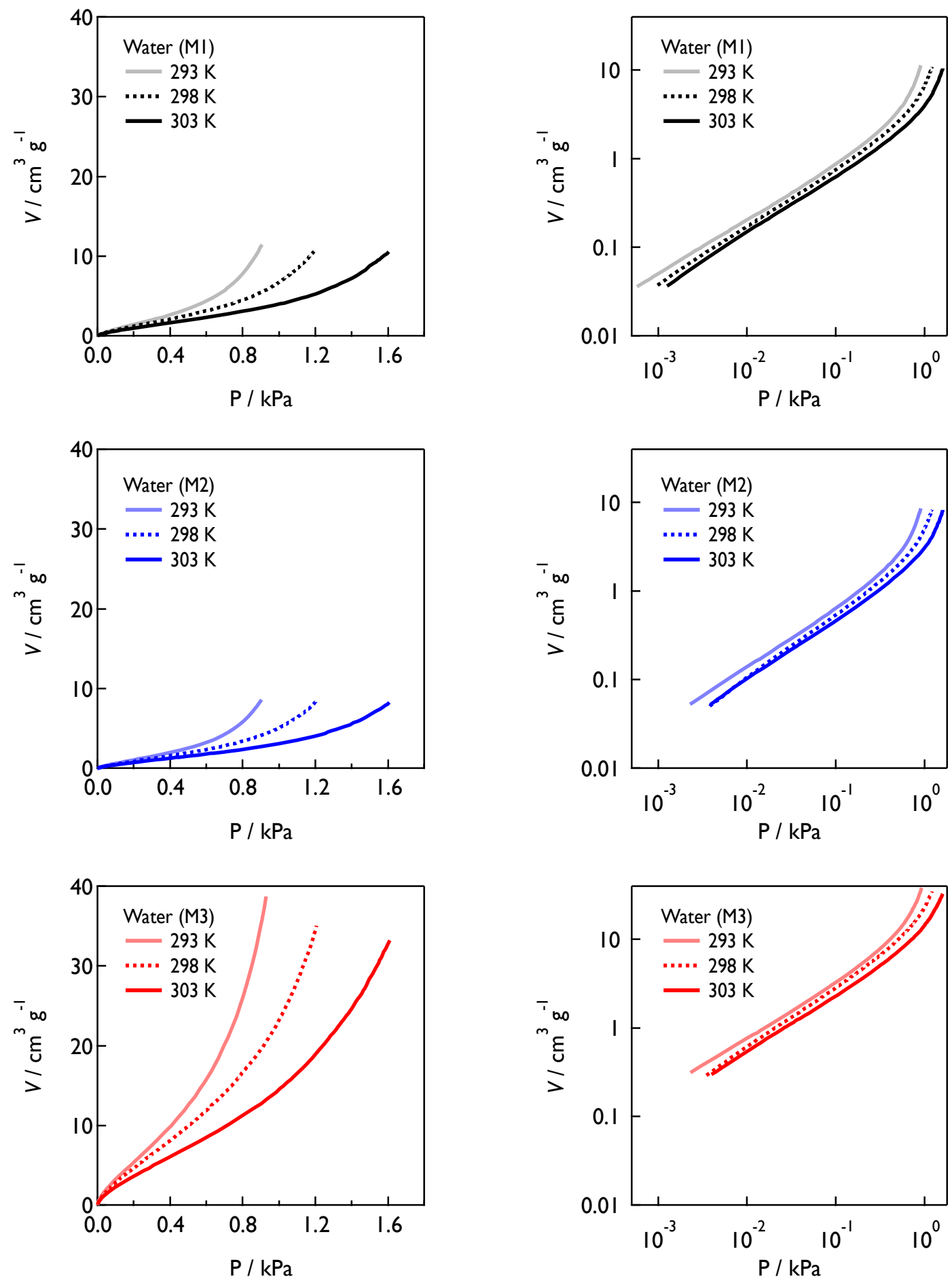

Fig. S2 Water adsorption isotherms for samples M1 (black), M2 (blue), and M3 (red) at 293, 298, and $303 \mathrm{~K}$. (left column) Axes are shown at a linear scale. (right column) Axes are shown at a logarithmic scale. 

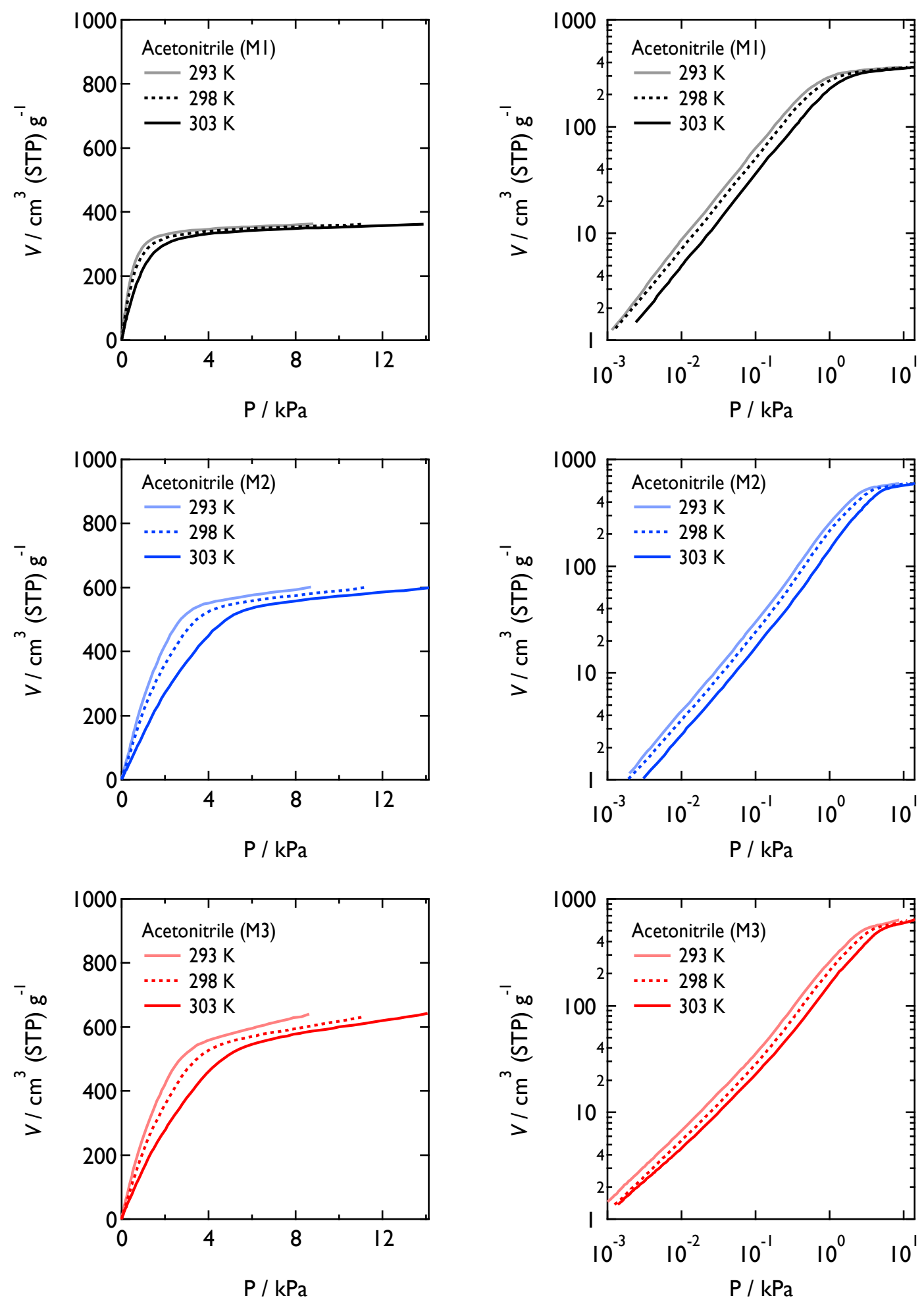

Fig. S3 Acetonitrile adsorption isotherms for samples M1 (black), M2 (blue), and M3 (red) at 293, 298, and 303 K. (left column) Axes are shown at a linear scale. (right column) Axes are shown at a logarithmic scale. 

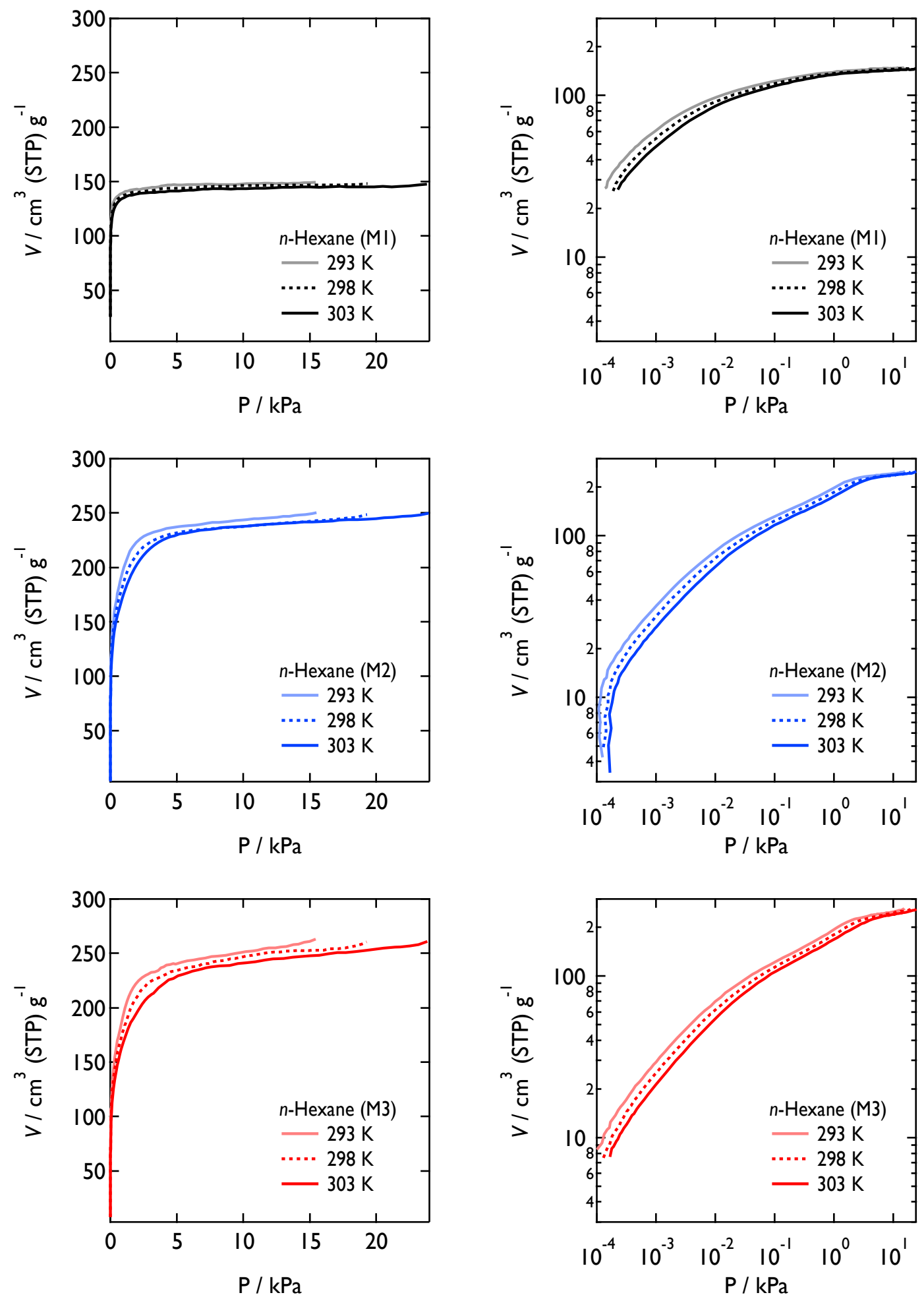

Fig. S4 $n$-hexane adsorption isotherms for samples M1 (black), M2 (blue), and M3 (red) at 293, 298, and $303 \mathrm{~K}$. (left column) Axes are shown at a linear scale. (right column) Axes are shown at a logarithmic scale. 
(a) Water
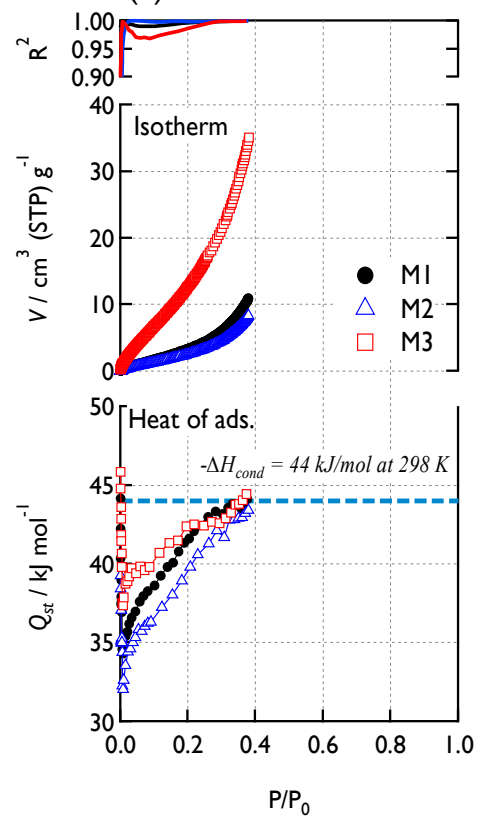

(b) Acetonitrile
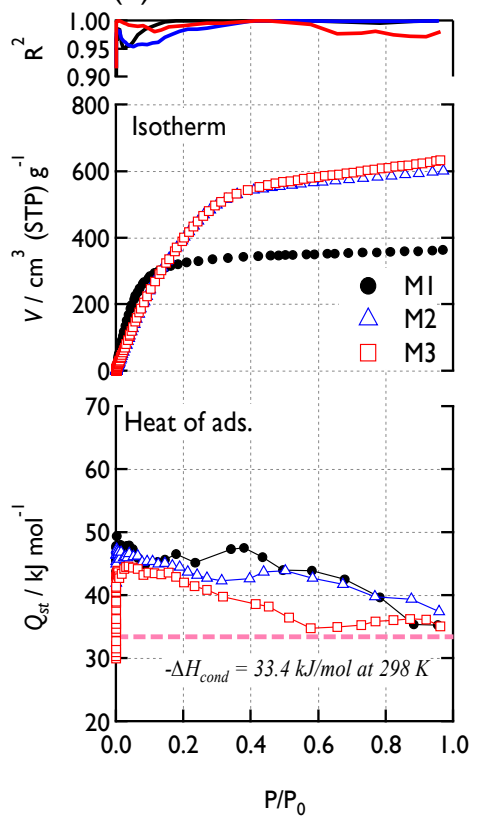

(c) Hexane
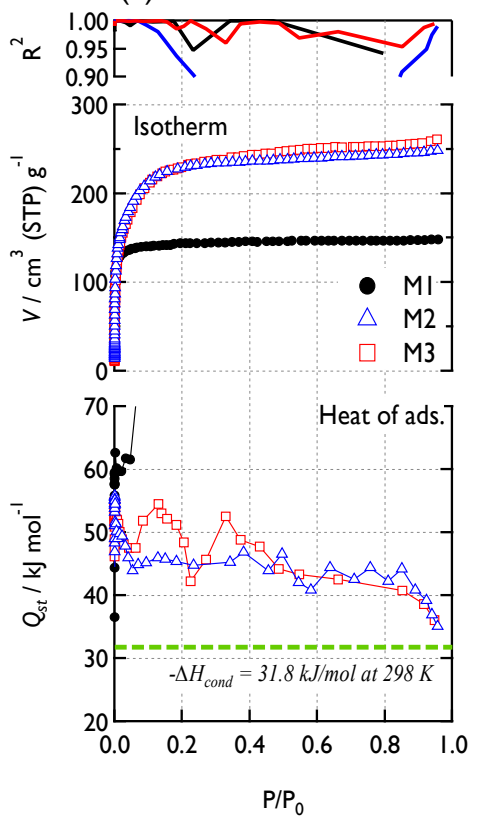

Fig. S5 Vapor adsorption isotherms at $298 \mathrm{~K}$ (middle), isosteric heat of adsorption (bottom), and coefficient of determination $\left(R^{2}\right)$ for applying the Clausius-Clapeyron equation (top) to samples M1 (black closed circle), M2 (blue open triangle), and M3 (red open square) for different adsorbents: (a) water, (b) acetonitrile, and (c) $n$-hexane. Broken lines in the bottom row represent the heat of condensation of each adsorbent.

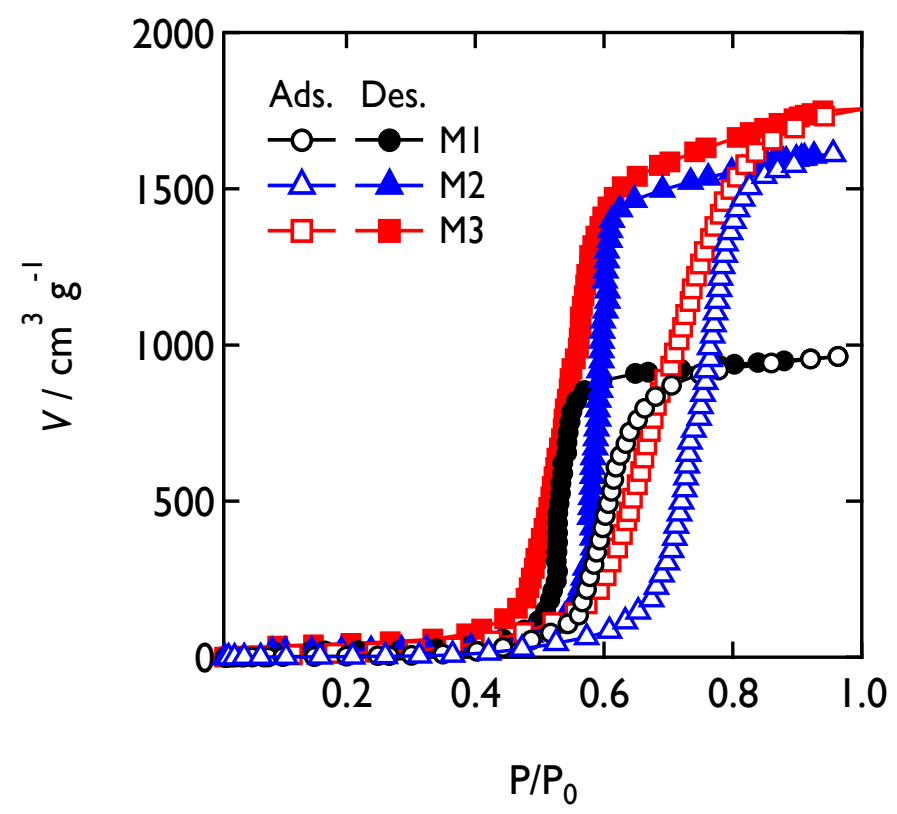

Fig. S6 Water adsorption isotherms for samples M1(black circle), M2 (blue triangle), and M3 (red square) at $298 \mathrm{~K}$ measured in the relative pressure range of 0-1.0. 